\title{
The Determination of the Sign of the Spin-Hamiltonian Parameters.
}

\author{
M. E. Fogrto \\ Centro Atómico Bariloche (C.N.E.A.) \\ Instituto de Fisica "Dr. José Antonio Balstiro" (U.N. de C. and O.N.E.A.) \\ San Carlos de Bariloche (Rio Negro) \\ (Nuovo Cimento, 50 B. $158(1967)$ )
}

Unfortunately two misprints have crept into the text of this letter.

On p. 159 , second column, line 10 , please read

instead of it is not possible to find the sign of $g_{x} g_{y} g_{z}$ it is possible to find the sign of $g_{x} g_{y} g_{z}$;

and on p. 160 , second column, line 21 , please read

instead of induced by $T_{4}$ included by $T_{4}$.

\section{Paramagnetic Susceptibility and Stability of the Electron Gas in a Modified Hartree-Fock Approximation.}

\author{
B. Johansson and K.-F. BergGRen \\ FOA - Stockholm \\ (Nuovo Cimento, $50 \mathrm{~B}, 362(1967))$
}

Equation (1) should read $v_{\mathrm{F}}=k_{\mathrm{F}} / \mathrm{m}^{*}$, and the third entry in the last column of Table I should be $H_{\mu_{0}}$. 